In mehr als jeder zweiten Beschwerde sei die mangelhafte Aufklärung über die Behandlungsalternativen auf Kasse kritisiert worden. Sollten die letzten beiden Angaben auch tatsächlich der Wahrheit entsprechen, so läge ein klarer Verstoß der Praxen gegen rechtliche Vorgaben vor.

Wie bereits kurz vor einem Jahr, so übt die VZ NRW weiter Kritik an sogenannten IGeL-Verzichtserklärungen. Jeder fünfte Patient sollte demnach aktuell bereits im Vorzimmer seine $\mathrm{Zu}$ stimmung oder Ablehnung einer Selbstzahlerleistung auf einem Formular ankreuzen und unterschreiben. „Die Verwendung solcher Verzichtserklärungen sind lediglich ein psychologisches Druckinstrument, das jeglicher rechtlichen Grundlage entbehrt. Solche Formulare sind unsinnig und gehören aus der Arztpraxis verbannt“, erklärt Schuldzinski.

\section{Verstöße werden abgemahnt}

Mit den Ophthalmologen und den Gynäkologen schneiden in der Beurteilung durch die Patienten nach dem Urteil der VZ NRW zwei Facharztgruppen besonders schlecht ab. Dermatologen tauchen dagegen mit Ausnahme der
Hautkrebsfrüherkennung so gut wie nicht auf. Die VZ NRW bemängelt allerdings, dass viele Hautärzte mit Kassenzulassung das gesetzliche Hautkrebsscreening ihren Patienten gar nicht erst anböten.

Nachweisliche Verstöße aufgrund von Forumsbeschwerden habe die VZ NRW bereits erfolgreich abgemahnt, wie sie betont. „Nun gilt es, die Patientenrechte zu stärken und die Ergebnisse für eine Verbesserung der Geschäftsbeziehungen von Patienten und Ärzten auf dem Gesundheitsmarkt zu nutzen“, postuliert Schuldzinski - garniert mit dem Hinweis der VZ NRW auf den teilweise umstrittenen medizinischen Nutzen einzelner Angebote. Dies stellt eine Art Selbstreferenz auf den vom Medizinischen Dienst des GKV-Spitzenverbandes etablierten - und von dem Verbraucherzentrale Bundesverband unterstützten - „IGeL-Monitor“ dar, bei dem nur wenigen Selbstzahlerangeboten aus wissenschaftlicher Sicht ein Nutzen zuerkannt wird (www.igel-monitor.de). Inzwischen haben sich schon mehrere Berufsverbände der betroffenen Fachgruppen zu Wort gemeldet und die pauschale Kritik an der IGeL-Praxis zurückgewiesen. Matthias Wallenfels

\title{
Praxisübernahme nur mit Sprechstundenbedarf
}

Wer die Praxis eines Kollegen übernehmen will, sollte auch den Sprechstundenbedarf des Vorgängers im Auge haben, um sich unangenehme Überraschungen zu ersparen. Er habe den Fehler gemacht, sich um das Thema Sprechstundenbedarf nicht zu kümmern, berichtete Dr. Mahmoud Delonge bei der Veranstaltung „Existenzgründung 2.0" der Deutschen Apothekerund Ärztebank, der ETL Steuerberatungsgesellschaft und der Ärzte Zeitung in Köln. „Ich habe einfach nicht gewusst, dass man am Anfang keinen Sprechstundenbedarf bestellen darf", so Delonge.

Bis auf Röntgenkontrastmittel und Impfstoffe müssen Ärzte zu Anfang ihrer vertragsärztlichen Tätigkeit den Sprechstundenbedarf selbst beschaffen.
Er gilt als Grundausstattung der Praxis. Die erstmalige Verordnung ist erst zum Ende des ersten Abrechnungsquartals möglich. Die KV Nordrhein weist darauf hin, dass bei einer Praxisübernahme der Vorgänger aber die zuletzt verbrauchten Materialien noch einmal als Sprechstundenbedarf bestellen kann. „Mein Vorgänger hatte nichts mehr, nicht einmal mehr Kompressen“, sagte Delonge. Das war teuer für ihn: Er musste $3.000 €$ selbst bezahlen.

Bei einer Praxisübernahme sei es grundsätzlich wichtig, ein ordentliches Inventarverzeichnis anzulegen, betonte Jens-Peter Jahn von der Anwaltskanzlei Dr. Halbe. „Man muss das Anlagenverzeichnis nehmen, durch die Praxis gehen und sehen, ob die Sachen da sind." Ilse Schlingensiepen 